\title{
Unit Commitment Solution Using Particle Swarm Optimisation (PSO)
}

\author{
Divya Ananthan ${ }^{1}$, Prof.S.Nishanthinivalli ${ }^{2}$ \\ ${ }^{I}$ (P.G Student, Jayaram college of Engineering \& Technology, Tiruchirapalli) \\ ${ }_{2}^{2}$ (Assistant Professor, Department of EEE, Jayaram College of Engineering and Technology, Tiruchirapalli)
}

\begin{abstract}
Existing unit commitment methods have the problem of stopping at local optimum and slow convergence. So it is replaced by a new method known as Particle Swarm Optimisation (PSO) which is a biological method based on particle swarming. It consists of a group of particles moving towards optimal solution. Feasible solutions are obtained as particles move in feasible solution space rather than infeasible ones. Thus the method reduces computational time. In this paper PSO is applied to IEEE 30 bus test system with six generators so that fuel cost of each generator is reduced using PSO.
\end{abstract}

Keywords: - $C_{p} C_{g}, G A, G_{b e s t}, K, P_{b e s t}, P S O, U C, v_{i}, W, x_{i} U_{i}$

\section{INTRODUCTION}

Due to the nature of changing technology, unit commitment is also undergoing a change in its solution method. This is because there must be an efficient method to commit the generators to meet the load. Many methods have been introduced to solve unit commitment. Even if the methods have advantages, most of the methods suffer from local convergence and curse of dimensionality. So a new method known as PSO is introduced to solve unit commitment.

The method was introduced by Eberhart and Kennedy in the year 1995.It is a biological based method based on the motion of particles in a hyperspace towards optimal solution. The project deals with the solution of unit commitment using PSO. Other applications of PSO are optimal placement of facts devices, reactive power dispatch, state estimation and fuzzy systems.

One such area is the commitment of thermal units. Particle swarm optimization has roots in biological simulation and behavior of birds and animals such as bird flocking, fish schooling, and swarming theory. It is also related to evolutionary computation, and has ties to both genetic algorithms and evolutionary programming.

Particle swarm optimization as developed by the authors comprises a very simple concept, and paradigms can be implemented in a few lines of computer code. It requires only primitive mathematical operators, and is computationally inexpensive in terms of both memory requirements and speed. Early testing has found the implementation to be effective with several kinds of problems.Particle swarm optimization has also been demonstrated to perform well on genetic algorithm test functions.

\section{PROBLEM FORMULATION}

Unit commitment can be defined as the selection of generators that must be operated to meet the forecasted load demand on the system over a period of time so that fuel cost is minimum $[2,3,4,5,6,7,8]$. The Unit Commitment Problem (UCP) is to determine a minimal cost turn-on and turn-off schedule of a set of electrical power generating units to meet a load demand [23] while satisfying a set of operational constraints.It is a well known problem in power industry and helps in saving fuel cost if units are committed correctly so that fuel cost is saved.

\subsection{NEED FOR UNIT COMMITMENT}

(i)Enough units will be committed to supply the load.

(ii)To reduce loss or fuel cost.

(iii)By running the most economic unit load can be supplied by that unit operating closer to its better efficiency.

\subsection{FACTORS CONSIDERED IN UNIT COMMITMENT}

(i) For finding the nature of fluctuating load as well as to commit the units accordingly a graph is drawn between load demand and hours of use. This graph is known as load curve. In the solution load pattern for M period is formed using load curve.

(ii)The possible numbers of units are committed to meet the load.

(iii)The load dispatch is calculated for all feasible combinations and operating limits of the units have to be calculated. 
Unit Commitment is considered as a complex optimization problem where the aim is to minimize the objective function in the presence of heavy constraints

The objective function is given by

Minimize Total cost $=$ Fuel cost + Start up cost + Shut down cost

Fuel cost: It is a quadratic function of power generated $\mathrm{Pg}$.

It is given by fuel cost $=\mathrm{a}_{\mathrm{i}} \mathrm{P}_{\mathrm{gi}}{ }^{2}+\mathrm{b}_{\mathrm{i}} \mathrm{P}_{\mathrm{gi}}+\mathrm{c}_{\mathrm{i}}$

Where $a_{i}, b_{i}$ and $c_{i}$ are the fuel cost coefficients of generator $i$ and $P_{g i}$ is the power generated by generator $i$.

Start up cost: When the unit is at rest, some energy is required to bring the unit online. This is the start up cost. It is maximum when the unit is at cold start (start up cost when cooling). The unit is given sufficient energy input to keep it at operating temperature(start up cost when banking).So it requires some energy input to the system to keep it at operating temperature.

Shut down cost: It is the cost for shutting down the unit. Sometimes during the shutdown period boiler may be allowed to cool down naturally and thus no shut down cost will be incurred.

\subsection{CONSTRAINTS IN UNIT COMMITMENT}

The constraints in unit commitment are $[1,2,3,4,11,15,16,17]$

(i) Load balance constraints: The real power generated must be able to meet the loads.

$$
\sum_{i=1}^{N} P g i=P_{D}
$$

(ii) Spinning reserve: It is the total power available from all units synchronised on the system minus present loads plus the losses. It is given by

$\sum_{i=1}^{N}\left(P_{\max i}-P_{g i}\right) U_{i} \geq \mathrm{SR}$

(iii) Thermal constraints: The temperature and pressure of units increase gradually as the units are started. So they must be synchronised before bringing online.

(iv) Must run units: Some of the units must be given a must-run status in order to provide voltage support for the network. For such units $U_{i}=1$.

(v) Power limits: Each generator has upper power limits and lower power limits within which the generators should run. It is given by

$$
P_{\text {gimin }} \leq P_{\text {gi }} \leq P_{\text {gimax }}
$$

(vi) Ramp rate constraints: The ramp rate constraint ensures that sufficient ramp rate capacity is committed to accommodate required generation changes. Any generation changes beyond the required changes are due strictly to economics of the committed generators.

(vii) Fuel constraints: The constraint means limited availability of fuel or burning of some amount of fuel.

\section{SOLUTION FOR THE OPTIMISATION PROBLEM}

The electric power industry has been using many methods to solve the unit commitment for decades. Due to complexity of the problem different solution methods are necessary [8].Reduction in computation time and fuel cost makes one method better than the other. In restructured market, small change in total cost can produce big changes in annual fuel cost.

A literature survey on unit commitment reveals that several methods have been developed to solve unit commitment $[1,2,3,4,5]$.They include

\subsection{DYNAMIC PROGRAMMING METHOD}

It is a stochastic search method which searches for solution from one state to the other. The feasible states are then saved $[1,11,19]$. Dynamic programming was the earliest optimization-based method to be applied to the UC problem. It is used extensively throughout the world. It has the advantage of being able to solve problems of a variety of sizes and to be easily modified to model characteristics of specific utilities[11].But the disadvantage of this method is curse of dimensionality. ie, the computational effort increases exponentially as problem size increases and solution is infeasible[4] and its suboptimal treatment of minimum up and downtime constraints and time-dependent startup costs[11].

\subsection{MIXED INTEGER LINEAR PROGRAMMING METHOD}

The method is widely used in the commitment of thermal units [2].It uses binary variables $(0$ or 1$)$ to represent start up, shut down and on/off status of units. It linearises the quadratic production cost into linear system and the start up cost into a staircase function. Even it guarantees optimal solution in finite number of steps, it fails when number of units increases because they require large memory space and suffer from great computational delay $[4,8]$. 


\section{3 LAGRANGE RELAXATION METHOD}

In this method the constraints are relaxed using Lagrange multipliers. A brief description of the method is given in [11].Unit commitment is written as a cost function involving a single unit and coupling constraints. Solution is obtained by adjoining coupling constraints and cost by Lagrange multipliers [11].[17] explains the scheduling of hydrothermal units using LA method. It provides a faster solution but solution feasibility and solution quality problem when number of units increases [4]. Lagrangian Relaxation is also being used regularly by some utilities [11]. Its utilization in production UC programs is much more recent than the dynamic programming.[19,24].But the disadvantage is that unit commitment obtained from an LR dual solution, even a "near-optimal" dual solution, usually displays over-commitment[35].

\subsection{FUZZY LOGIC METHOD}

Zadeh introduced the concept of fuzzy sets in 1965 as a mathematical means of describing vagueness in linguistics. It was later developed by mathematical researchers in 1970[11]. The idea may be considered as a generalization of classical set theory. UC is a complex decision-making process [1,3,5]which operates appropriate units at different hours and schedules the outputs of the committed units to meet a predicted demand, such that the operating cost is minimized. Due to the uncertainty of the demand and outages of generating units, fuzzy is used to represent the uncertainty. The method is an intelligence based technique that quantifies linguistic terms so that variables are treated as continuous. It establishes the relation between input and output according to some fuzzy control rules.eg by using "if-then". The result is defuzzified to obtain numerical solution [4].But the disadvantage of this method is that it cannot handle large scale systems [5].

\subsection{SIMULATED ANNEALING}

SA is a recently developed optimization technique, proposed by Kirkpatrick, Gelatt, and Vecchi in 1983, which takes advantage of the analogy between the minimization of the cost function of an optimization problem and the slow procedure of gradually cooling a metal, until it reaches its "freezing" point, where the energy of the system has acquired the globally minimal value [11,32]. The algorithm is based on the iterative method which simulates the transition of atoms in equilibrium at a given temperature. SA serves for solving difficult combinatorial optimization problems without specific structure. The main drawback of this method is that it requires long CPU time, due to the large number of iterations needed for the convergence of the algorithm.

\section{6. GENETIC ALGORITHM}

It consists of an initial member of population. It is a matrix of number of generators and time of scheduling. The generators are given status of 0 or 1 . Most highly fit members in a population are selected to pass on information to the next population of members [12]. A crossover point is then selected at random and information from one parent, up to the crossover point, exchanged with the other member thus creating two new members for the next generation. The better performing members are rewarded well [20].The benefits of using a genetic algorithm (GA) are: a robust optimization technique, easy implementation and production of multiple UC schedules.

They operate on a population of potential solutions applying the principle of survival of the fittest to produce successively better approximations to a solution [27].GA has also been applied for solving the UCP of hydrothermal power system.

\section{EVOLUTION OF PSO}

Since all the previous methods suffer from dimensionality and computation problems, a new method has been evolved in solving the unit commitment. It is known as Particle Swarm Optimisation method.[6,15,21].The method was developed [13] by simulation of social model. The method is inspired from social behaviour such as "bird flocking" or "fish schooling". Concept of bird flocking was introduced by Reynolds. Later Hepner modelled group motion, disintegration and changing direction of group.

\subsection{CONCEPT OF PSO}

The method consists of a group of particles in a given dimension moving towards optimal solution. The particles move based on their previous best position, the position of neighbours and the best among all particles[15,33].Each particle move towards the optimal solution based on its previous best position given by $P_{b e s t}$, position of other particles and the best among all the other particles given by $G_{b e s t}$. The search is continued until a globally best solution is obtained or specific number of iteration is reached. 


\subsection{SOLUTION METHODOLOGY:}

Consider a system having $\mathrm{N}$ particles moving in $\mathrm{D}$ dimensional space. Let the position of $\mathrm{i}_{\mathrm{th}}$ particle be $\mathrm{x}_{\mathrm{i}}$ and its velocity be $\mathrm{V}_{\mathrm{i}}$. When the particle move from one position to the other, its velocity is updated using the rule,

$V_{i}^{k+1}=\mathrm{W} V_{i}^{k}+C_{p}{ }^{*} \operatorname{rand} 1\left(P_{\text {besti }}^{k}-x_{i}^{k}\right)+C_{g}{ }^{*} \operatorname{rand} 2\left(G_{\text {best }}^{k}-x_{i}^{k}\right)$

Where,

$\mathrm{W}$ is the inertia weight.

$C_{p}$ and $C_{g}$ are acceleration constants which accelerate the particle towards $P_{\text {best }}$ and $G_{\text {best }}$ respectively.k is the iteration number.

rand 1 and rand 2 are random numbers of the range 0 to 1 .

The equation for $\mathrm{W}$ is given by

$\mathrm{W}=W_{\text {final }}+\frac{k_{\max }-k}{K_{\max }}\left(W_{\text {initial }}-W_{\text {final }}\right)$

Where,

$W_{\text {initial }}=$ initial inertia weight.

$W_{\text {final }}=$ final inertia weight.

$K_{\max }=$ final inertia weight.

Value of $\mathrm{W}$ is varied between 0.9 and 0.4 to find optimal solution.

Later the position of the particle is updated using the rule,

$x_{i}^{k+1}=x_{i}^{k}+V_{i}^{k+1}$

\subsection{PSO ALGORITHM} algorithm.

According to the formulation above, the following procedure can be used for implementing the PSO

1) Initialize the swarm by assigning a random position in the problem hyperspace to each particle.

2) Evaluate the fitness function for each particle

3) For each individual particle, compare the particle's fitness value with its $P_{\text {best }}$. If the current value is better than the $P_{\text {best }}$ value, then set this value as the $P_{\text {best }}$ and the current particle's position, $\mathrm{x}_{\mathrm{i}}$ as $P_{i}$.

4) Identify the particle that has the best fitness value. The value of its fitness function is identified as $G_{b e s t}$ and its position as $P_{g}$.

5) Update the velocities and positions of all the particles using (1) and (2).

6) Repeat steps 2-5 until a stopping criterion is met (e.g., maximum number of iterations or a sufficiently good fitness value)

\subsection{ADVANTAGES OF PSO COMPARED TO CONVENTIONAL METHODS:}

1. Easy to implement and potential to achieve a high quality solution with stable convergence characteristics.

2. The particles are treated as volume less and each particle update position and velocity according to its own experience and partners experience. [8]

3. PSO is more capable of maintaining diversity of the swarm.

4. One of reasons that PSO is attractive is that there are very few parameters to adjust [21]

\subsection{PSO CONCEPTS:}

The basic concepts of PSO are classified as

1. Social concepts: Human intelligence is based on social interaction, evolution, comparison and imitation from others.

2. Swarm Intelligence Principle: It has five fundamental principles

(i)Proximity principle: Particle should carry simple space and time computation.

(ii)Quality principle: Particle should respond to quality factors in environment.

(iii)Diverse response principle: Particle should not commit its activity in extensively narrow channel.

(iv)Stability principle: Particle should not commit its activity in extensively narrow channels.

(v)Adaptability principle: Particle should change its behaviour when worth computational.

\subsection{APPLICATIONS OF PSO}

PSO has found great applications in many fields such as unit commitment, economic dispatch, power quality, reactive power control, voltage security etc.The important two applications are scheduling of pumped storage plant [9] and electromagnetics [10].Other applications are human tremor analysis, power system load stabilisation and product mix optimisation [32] 
The pumped storage unit generates at peak load and pumps at light load. It uses a PSO method and a mutation operation to find solution. The electromagnetic problem uses a robust PSO where particles are arranged in ascending order according to feasibility and energy is reduced.

\section{RESULT}

In this paper PSO is applied on IEEE 30 bus system. It consists of 6 generators as shown in figure.

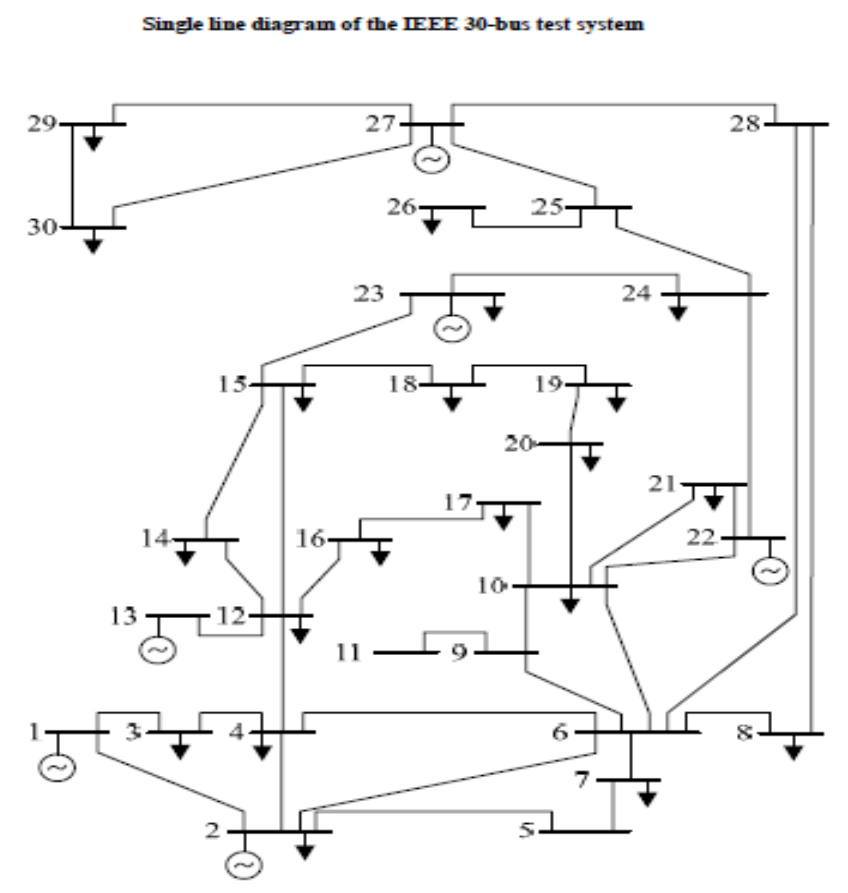

Fig 1

The generator data is given in table below

\begin{tabular}{|c|c|c|c|c|c|c|c|c|}
\hline \multicolumn{9}{|c|}{ Generator data of IEEE 30 bys system } \\
\hline \multirow{2}{*}{ UNIT } & \multirow{2}{*}{ BUS } & \multicolumn{3}{|c|}{ COST COEFFICIENT } & \multirow{2}{*}{ PMAX } & \multirow{2}{*}{ PMIN } & \multirow{2}{*}{$\begin{array}{l}\text { MIN UP } \\
\text { TIME }\end{array}$} & \multirow{2}{*}{$\begin{array}{c}\text { MIN } \\
\text { DOWN } \\
\text { TIME }\end{array}$} \\
\hline & & $\mathbf{A}$ & B & $\mathbf{C}$ & & & & \\
\hline 1 & 1 & 0.02 & 15 & 0 & 80 & 15 & 2 & 2 \\
\hline 2 & 2 & 0.0175 & 14.75 & 0 & 80 & 15 & 2 & 2 \\
\hline 3 & 13 & 0.025 & 16 & 0 & 50 & 10 & 3 & 3 \\
\hline 4 & 22 & 0.0625 & 14 & 0 & 50 & 10 & 4 & 4 \\
\hline 5 & 23 & 0.025 & 16 & 0 & 30 & 5 & 3 & 3 \\
\hline 6 & 27 & 0.0083 & 15.25 & 0 & 55 & 10 & 4 & 4 \\
\hline
\end{tabular}

The PSO algorithm is applied to IEEE 30 bus system considering three random particles. Each particle undergoes 10 iterations. The minimum value of fuel cost as well as the power generated at minimum fuel cost is calculated and tabulated below.

A Priority list of Units are created according to the input data and the units are turned on as shown in table below.

\begin{tabular}{|c|l|c|c|}
\hline Ranking Order & \multicolumn{1}{|c|}{ Unit } & L (Mw) & U (Mw) \\
\hline 1 & 6 & 10 & 55 \\
\hline 2 & $6+2$ & 25 & 135 \\
\hline 3 & $6+2+1$ & 40 & 215 \\
\hline 4 & $6+2+1+5$ & 45 & 245 \\
\hline 5 & $6+2+1+4+5$ & 55 & 295 \\
\hline 6 & $6+2+1+5+4+3$ & 65 & 345 \\
\hline
\end{tabular}

Where $\mathrm{L}$ is Lower bound of units and $\mathrm{U}$ is upper bound of units. 
The table shows the turn on and turn off status of units.

\begin{tabular}{|l|l|l|l|l|l|l|}
\hline \multirow{2}{*}{ Time } & \multicolumn{7}{|c|}{ Unit Status } \\
\cline { 2 - 7 } & 1 & 2 & 3 & 4 & 5 & 6 \\
\hline 1. & 1 & 1 & 0 & 0 & 0 & 1 \\
\hline 2. & 1 & 1 & 0 & 0 & 1 & 1 \\
\hline 3. & 1 & 1 & 1 & 1 & 1 & 1 \\
\hline 4. & 1 & 1 & 1 & 1 & 1 & 1 \\
\hline 5. & 1 & 1 & 1 & 1 & 1 & 1 \\
\hline 6. & 1 & 1 & 1 & 1 & 1 & 1 \\
\hline 7. & 1 & 1 & 0 & 1 & 1 & 1 \\
\hline 8. & 1 & 1 & 0 & 1 & 1 & 1 \\
\hline 9. & 1 & 1 & 0 & 0 & 1 & 1 \\
\hline 10. & 1 & 1 & 0 & 0 & 0 & 1 \\
\hline 11. & 1 & 1 & 0 & 0 & 0 & 1 \\
\hline 12. & 1 & 1 & 0 & 0 & 0 & 1 \\
\hline 13. & 1 & 1 & 0 & 0 & 0 & 1 \\
\hline 14. & 1 & 1 & 0 & 0 & 1 & 1 \\
\hline 15. & 1 & 1 & 0 & 1 & 1 & 1 \\
\hline 16. & 1 & 1 & 0 & 1 & 1 & 1 \\
\hline 17. & 1 & 1 & 0 & 1 & 1 & 1 \\
\hline 18. & 1 & 1 & 0 & 1 & 1 & 1 \\
\hline 19. & 1 & 1 & 0 & 1 & 1 & 1 \\
\hline 20. & 1 & 1 & 0 & 1 & 1 & 1 \\
\hline 21. & 1 & 1 & 0 & 0 & 1 & 1 \\
\hline 22. & 1 & 1 & 0 & 0 & 0 & 1 \\
\hline 23. & 1 & 1 & 0 & 0 & 0 & 1 \\
\hline 24. & 1 & 1 & 0 & 0 & 0 & 1 \\
\hline
\end{tabular}

The PSO algorithm is applied to above system considering three random particles. Each particle undergoes 10 iterations. The Spinning Reserve is taken as $10 \%$ of maximum demand. The minimum value of fuel cost as well as the power generated at minimum fuel cost is calculated and tabulated below.

\begin{tabular}{|c|c|c|c|c|c|c|}
\hline Load Demand & G1 & G2 & G3 & G4 & G5 & G6 \\
\hline 166 & 66.0251 & 72.6845 & - & - & - & 54.6221 \\
\hline 196 & 66.0251 & 72.6845 & - & - & 29.8964 & 54.6221 \\
\hline 229 & 61.55 & 70.406 & 38.93 & 36.716 & 29.8945 & 54.577 \\
\hline 267 & 61.55 & 70.406 & 38.93 & 36.716 & 29.8945 & 54.577 \\
\hline 283.4 & 68 & 73.7 & 42.8 & 41.36 & 29.8985 & 54.6899 \\
\hline 272 & 61.55 & 70.406 & 38.93 & 36.716 & 29.8945 & 54.577 \\
\hline 246 & 68 & 73.7 & - & 41.36 & 29.8985 & 54.6899 \\
\hline 213 & 55.2768 & 67.1397 & - & 32.1156 & 29.8926 & 54.4719 \\
\hline 192 & 61.55 & 70.406 & - & - & 29.8945 & 54.577 \\
\hline 161 & 61.55 & 70.406 & - & - & - & 54.577 \\
\hline 147 & 53.5938 & 66.2687 & - & - & - & 54.4630 \\
\hline 160 & 61.55 & 70.406 & - & - & - & 54.577 \\
\hline 170 & 68 & 73.7 & - & - & - & 54.6899 \\
\hline 185 & 75 & 77.4 & - & - & - & 54.8 \\
\hline 208 & 74.0605 & 76.8352 & - & - & 29.9081 & 54.7259 \\
\hline 232 & 61.55 & 70.406 & - & 36.716 & 29.8945 & 54.577 \\
\hline 246 & 68 & 73.7 & - & 41.36 & 29.8985 & 54.6899 \\
\hline 241 & 68 & 73.7 & - & 41.36 & 29.8985 & 54.6899 \\
\hline 236 & 66.0251 & 72.6845 & - & 39.8867 & 29.8964 & 54.6221 \\
\hline 225 & 61.55 & 70.406 & - & 36.716 & 29.8945 & 54.577 \\
\hline 204 & 68 & 73.7 & - & - & 29.8985 & 54.6899 \\
\hline 182 & 75 & 71.4 & - & - & - & 54.8 \\
\hline 161 & 61.55 & 70.406 & - & - & - & 54.577 \\
\hline 131 & 52 & 52 & - & - & - & 55 \\
\hline & & & & & & \\
\hline
\end{tabular}


The total fuel cost obtained using this method is Rs. 91281/- The results are compared with Lambda Iteration Method and following schedule is obtained.

\begin{tabular}{|c|c|c|c|c|c|c|c|}
\hline $\begin{array}{c}\text { Load } \\
\text { Demand }\end{array}$ & $\lambda$ Value & G1 & G2 & G3 & G4 & G5 & G6 \\
\hline 166 & 17.4613 & 61.5239 & 77.4662 & - & - & - & 55 \\
\hline 196 & 17.4718 & 61.7961 & 77.767 & - & - & 29.4369 & 55 \\
\hline 229 & 17.5476 & 63.6891 & 79.9304 & - & 28.3805 & 30 & 55 \\
\hline 267 & 17.6792 & 66.9811 & 80 & 33.5849 & 29.434 & 30 & 55 \\
\hline 283.4 & 17.9887 & 74.7170 & 80 & 39.7736 & 31.9094 & 30 & 55 \\
\hline 272 & 17.7736 & 69.3396 & 80 & 35.4717 & 30.1887 & 30 & 55 \\
\hline 246 & 18.0606 & 76.5151 & 80 & - & 32.4848 & 30 & 55 \\
\hline 213 & 17.3398 & 58.4939 & 73.993 & - & 26.7180 & 26.7951 & 55 \\
\hline 192 & 17.3335 & 60.4369 & 76.2136 & - & - & 28.3495 & 55 \\
\hline 161 & 17.368 & 59.2 & 74.8 & - & - & - & 55 \\
\hline 147 & 17.1067 & 52.6667 & 67.3333 & - & - & - & 55 \\
\hline 160 & 17.3493 & 58.7333 & 74.2667 & - & - & - & 55 \\
\hline 170 & 17.5360 & 63.4 & 79.6 & - & - & - & 55 \\
\hline 185 & 18.12 & 80 & 80 & - & - & - & 55 \\
\hline 208 & 17.84 & 71 & 80 & - & - & 30 & 55 \\
\hline 232 & 17.6364 & 65.9091 & 80 & - & 29 & 30 & 55 \\
\hline 246 & 18.0606 & 76.5151 & 80 & - & 32.4848 & 30 & 55 \\
\hline 241 & 17.9091 & 72.7273 & 80 & - & 31.2727 & 30 & 55 \\
\hline 236 & 17.7576 & 68.9394 & 80 & - & 30.0606 & 30 & 55 \\
\hline 225 & 17.4869 & 62.1716 & 78.1961 & - & 27.8949 & 29.7373 & 55 \\
\hline 204 & 17.68 & 67 & 80 & - & - & 30 & 55 \\
\hline 182 & 18 & 75 & 80 & - & - & - & 55 \\
\hline 161 & 17.368 & 59.2 & 74.8 & - & - & - & 55 \\
\hline 131 & 16.9096 & 50 & 64.2857 & - & - & - & 55 \\
\hline
\end{tabular}

The fuel cost obtained using this method is Rs.91923/- it shows that PSO method is more efficient than Lambda Iteration Method because it reduces the fuel cost.

Simulations are performed in mat lab software and a graph is drawn between number of iterations and fuel cost. The graph obtained is exponentially decreasing showing convergence. The graph is shown below.

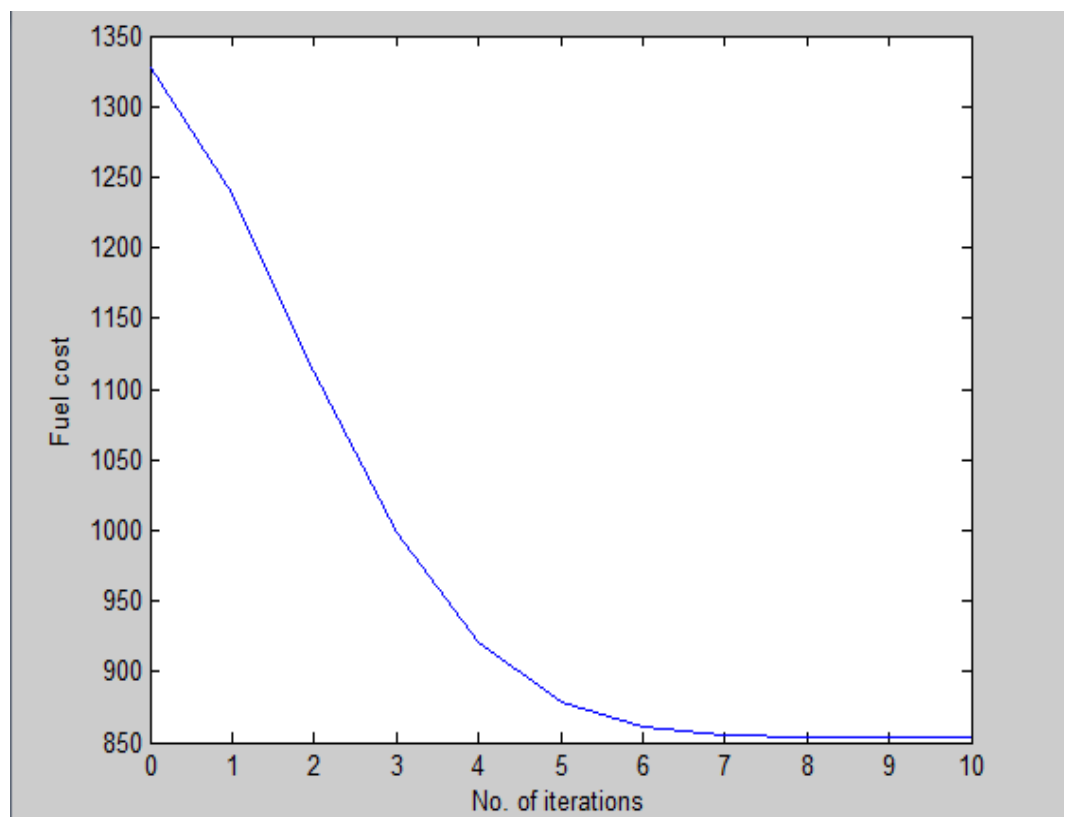

The graph is drawn between velocity and no.of iterations. The graph shows that velocity increases at the beginning for fast convergence and later reaches the constant value showing convergence. 


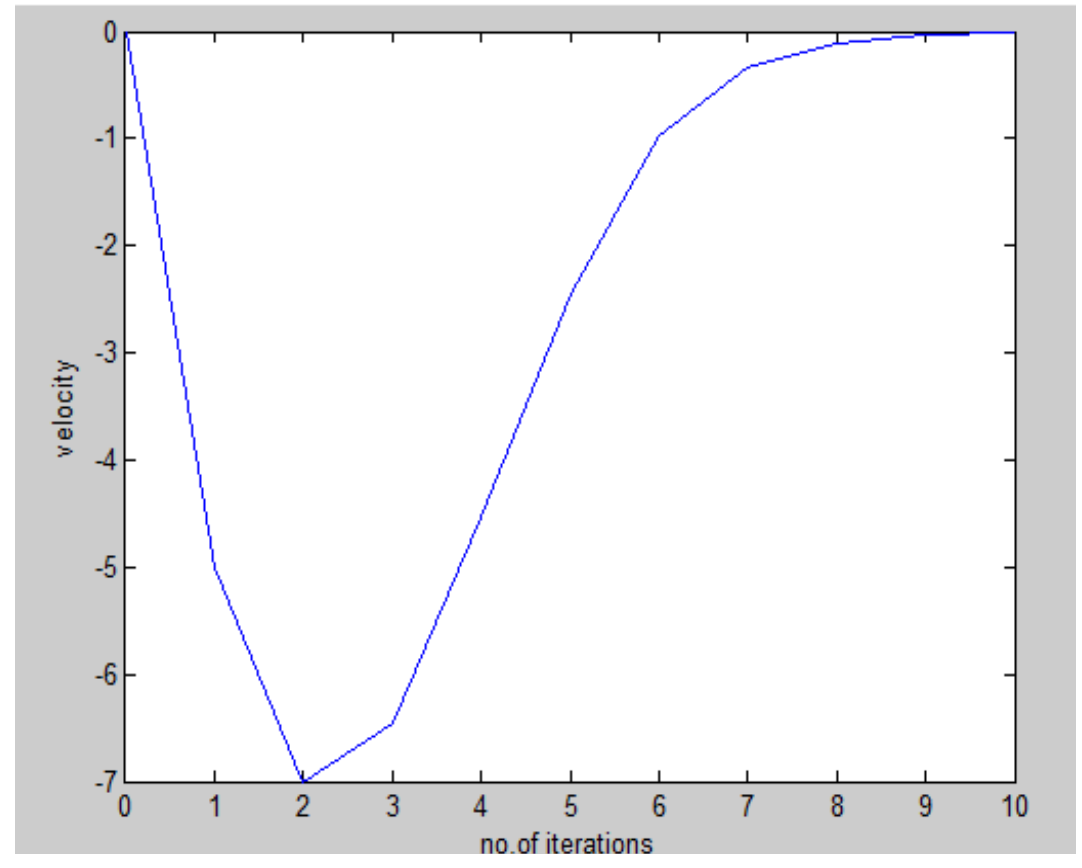

VI. CONCLUSION

PSO method is applied to IEEE 30 bus system and the fuel cost is reduced. The result is compared with Lambda Iteration Method showing the efficiency of PSO Method. The method can also be extended to above 30 bus system. The graph obtained also shows convergence.

\section{REFERENCES}

[1] Alam M.S, El-Sharkh M.Y,Rahman A and, Sisworahardjo.N. S "An Improved Ant Colony Search Algorithm for Unit Commitment Application" IEEE transactions on Power Systems Vol6,No.2 February 2006.

[2] Alice E Smith and Jorge Valenzuela "A seeded Memetic algorithm for large unit commitment problem" Journal of Heuristics October 2000.

[3] Alva J. Svoboda (Member, IEEE ),Chao-an Li,Chung-Li Tseng, Eric Hsu and Raymond B. Johnson "A robust unit commitment algorithm for hydro-thermal optimization" IEEE Transactions on Power Systems, Vol. 13, No. 3, August 1998.

[4] Amit Jain,Lingamurthy Belede, and Ravikanth Reddy Gaddam "Unit Commitment with Nature and Biologically Inspired Computing" Power Systems Research Center IIIT Hyderabad, India, 2009 World Congress on Nature \& Biologically Inspired Computing (NaBIC 2009).

[5] Angelina Jane Reyes-Medina, Gregorio Toscano-Pulido, Jos'e Gabriel and Ram'irez-Torres "A Statistical Study of the Effects of Neighborhood Topologies in Particle Swarm Optimization" Computational Intelligence,Springer 2011.

[6] Arthur I Cohen and Gregory Ostrowski "Scheduling units with multiple operating modes in unit commitment"IEEE Transactions on Power Systems, Vol 11,No.1,February 1996.

[7] Beltran.C and Heredia.F.J "Unit Commitment by Augmented Lagrangian Relaxation: Testing Two Decomposition Approaches" Journal of Optimization Theory and Applications: vol. 112, No. 2, February 2002

[8] Carlos Murillo and Robert $\mathrm{J}$ Thomas "Thermal unit commitment with non linear power flow constraints"1998 IEEE.

[9] Chung Li an Raymond B Johnson "A transmission constrained unit commitment method"1998 IEEE.

[10] Chenthur Pandian.S, and Dr. Duraiswamy.K "Fuzzy Logic Implementation for Solving the Unit Commitment Problem"IEEE 2004 International Conference on Power System Technology POWERCON 2004 Singapore, 21-24 November 2004.

[11] Claus c. caroe and Rudiger Schultz "A Two-Stage Stochastic Program for Unit Commitment Under Uncertainty in a Hydro-Thermal Power System" University of Copenhagen,February 1998

[12] Costas D. Vournas, Dimitris N. Simopoulos and Stavroula D. Kavatza "Unit Commitment by an Enhanced Simulated Annealing Algorithm" PSCE 2006.

[13] Dipangar Dasgupta "Unit commitment in thermal power generation using genetic algorithm"Sixth International Conference of Industrial Engineering and application of artificial systems June 1993 
[14] Dipti Srinivasan and Logentiran T "Particle Swarm Optimization for Unit Commitment Problem" IEEE Transactions on Power Systems, Vo16, No. 2, May 2010

[15] Emmanuel S. KARAPIDAKIS and Yiannis A. KATSIGIANNIS "Comparing different approaches to solve the unit commitment problem considering hydropumped storage stations"Dogus University,DEMSEE 2007.

[16] Erik.J, John R Birge and Samer Takriti "Intelligent unified control of unit commitment"University of Michigan.technical report,September 1994

[17] Erlich.I , Senior Member, IEEE and Pappala V.S, Student Member, IEEE, "A New Approach for Solving the Unit Commitment Problem by Adaptive Particle Swarm Optimization" 2008 IEEE

[18] Feng Luan, Jong-Ho Choi and Hyun-kyo-Jung "A Particle Swarm Optimisation Algorithm with Novel Expected Fitness Evaluation for Robust Optimisation Problems". IEEE transactions on Magnetics Volume 48, No.2 February 2012

[19] Ganesh Kumar Venayagamoorthy, Jean-Carlos Hernandez, Ronald G. Harley,Salman Mohagheghi and Yamille del Valle "Particle Swarm Optimization: Basic Concepts, Variants and Applications in Power Systems" IEEE Transactions on Evolutionary Computation, Vo1.12, No. 2, April 2008

[20] Gerald B. Sheble and Tim T. Maifeld "Genetic-based unit commitment algorithm" IEEE Transactions on Power Systems, Vol.11, No. 3, August 1996.

[21] Joon-Hyung Park,Sun-Kyo Kim, Geun-Pyo Park, Sang-Seung Le and Yong-Tae Yoon and Modified Dynamic Programming Based Unit Commitment Technique" IEEE Transactions on Power Systems, Vol. 10, No. 2, March 2010

[22] Houzong Yan and Peter M Rogan "Scheduling of Hydrothermal Power Systems"IEEE transactions on Power System, Vol.8, No.3, August 1993

[23] Huseyin hakan balci and Jorge F. Valenzuela "Scheduling electric power generators using particle swarm optimization combined with the lagrangian relaxation method "International Journal for Applied Mathematics and Computer Science Vol.14,No.3,May 2004.

[24] Irissari.K,Mokhtari.S and Wang.S.J "Short term generation scheduling with transmission and environmental constraints with an augmented lagrange relaxation"IEEE Transactions on Power Systems, Vol.10,N0.3,August 1995

[25] James Kennedy' and Russell Eberhart "Particle Swarm Optimization"1995 IEEE

[26] João Catalão, Sílvio Mariano and Victor Mendes "Unit commitment with environmental considerations: a practical approach" 15th PSCC, Liege, 22-26 August 2005

[27] Jonathan F Bard "Short term scheduling of thermal electric generators via Lagrange Relaxation "Operations Research Vol.36, No.5,September 1988

[28] José M. Arroy and Miguel Carrion "A Computationally Efficient Mixed-Integer Linear Formulation for the Thermal Unit Commitment Problem" IEEE Transactions on Power Systems, Vol. 21, No. 3, August 2006.

[29] Narayana Prasad Padhy "Unit Commitment-A Bibliographical Survey" IEEE transactions on power systems, vol. 19, No. 2, May 2004

[30] Po Hung Chen "Pumped-Storage Scheduling Using Evolutionary Particle Swarm Optimization" IEEE Transactions on Energy Conversion, Vol. 23, No. 1, March 2008

[31] Qiaozhu Zhai, Wei Fan and Xiaohong Guan and "A new method for unit commitment with ramping constraints" Electric Power Systems Research(ELSIVIER) February 2002.

[32] Russel Eberheart and Yuhui Shi "Particle Swarm Optimisation-development,application and resources"2001 IEEE

[33] Russell Eberhart and Yuhui Shi "A Modified Particle Swarm Optimizer"1998 IEEE

[34] Steven R. Braymer and William L Peterson "A Capacity Based Lagrangian Relaxation Unit Commitment with Ramp Rate Constraints" IEEE Transactions on Power Systems, Vol. 10, No. 2, May 1995

[35] Wen-Jun Zhang ,Xiao-Feng Xie and Zhi-Lian Yang "A Dissipative Particle Swarm Optimization"Congress on Evolutionary Computation Hawai,USA 2002. 